\title{
Shorter Chain Triglycerides Are Negatively Associated with Symptom Improvement in Schizophrenia
}

\author{
Anna Tkachev ${ }^{1, *}$, Elena Stekolshchikova ${ }^{1}$, Nickolay Anikanov ${ }^{1}$, Svetlana Zozulya ${ }^{2}$, Aleksandra Barkhatova ${ }^{2}$, \\ Tatiana Klyushnik ${ }^{2}$ and Daria Petrova ${ }^{1}$ i \\ 1 V. Zelman Center for Neurobiology and Brain Restoration, Skolkovo Institute of Science and Technology, \\ 121205 Moscow, Russia; E.Stekolschikova@skoltech.ru (E.S.); N.Anikanov@skoltech.ru (N.A.); \\ D.Petrova@skoltech.ru (D.P.) \\ 2 Mental Health Research Center, 115522 Moscow, Russia; s.ermakova@mail.ru (S.Z.); \\ abarkhatova@yandex.ru (A.B.); klushnik2004@mail.ru (T.K.) \\ * Correspondence: anna.tkachev@skolkovotech.ru
}

\section{check for}

updates

Citation: Tkachev, A.;

Stekolshchikova, E.; Anikanov, N.;

Zozulya, S.; Barkhatova, A.;

Klyushnik, T.; Petrova, D. Shorter

Chain Triglycerides Are Negatively

Associated with Symptom

Improvement in Schizophrenia.

Biomolecules 2021, 11, 720. https:/ /

doi.org/10.3390/biom11050720

Academic Editor: Cecilia M.

P. Rodrigues

Received: 18 April 2021

Accepted: 8 May 2021

Published: 11 May 2021

Publisher's Note: MDPI stays neutral with regard to jurisdictional claims in published maps and institutional affiliations.

Copyright: (C) 2021 by the authors. Licensee MDPI, Basel, Switzerland. This article is an open access article distributed under the terms and conditions of the Creative Commons Attribution (CC BY) license (https:/ / creativecommons.org/licenses/by/ $4.0 /)$.

\begin{abstract}
Schizophrenia is a serious mental disorder requiring lifelong treatment. While medications are available that are effective in treating some patients, individual treatment responses can vary, with some patients exhibiting resistance to one or multiple drugs. Currently, little is known about the causes of the difference in treatment response observed among individuals with schizophrenia, and satisfactory markers of poor response are not available for clinical practice. Here, we studied the changes in the levels of 322 blood plasma lipids between two time points assessed in 92 individuals diagnosed with schizophrenia during their inpatient treatment and their association with the extent of symptom improvement. We found 20 triglyceride species increased in individuals with the least improvement in Positive and Negative Syndrome Scale (PANSS) scores, but not in those with the largest reduction in PANSS scores. These triglyceride species were distinct from the rest of the triglyceride species present in blood plasma. They contained a relatively low number of carbons in their fatty acid residues and were relatively low in abundance compared to the principal triglyceride species of blood plasma.
\end{abstract}

Keywords: schizophrenia; lipid; lipidomics; triglyceride; mass spectrometry; PANSS

\section{Introduction}

Psychiatric disorders, including schizophrenia (SCZ), can be debilitating disorders that, if left untreated, give rise to severe problems that affect every area of life. While antipsychotics, among other possible additional treatments, are typically used as medication aimed to reduce symptoms of schizophrenia, treatment efficacy varies between individuals [1], and it is estimated that up to $34 \%$ of SCZ patients exhibit resistance to two or more antipsychotic medications [2]. Satisfactory biomarkers of treatment response and treatment resistance in schizophrenia would be extremely valuable, but are yet to be discovered [3]. Such biomarkers would not only be promising for personalized treatment plans, but also, possibly, provide fundamental information on the pathophysiology of the disorder.

Lipidomics in psychiatric disorders is an area of research gaining traction during the past years. In part, this can be attributed to the fast-developing field of lipid research [4,5]. There is also a growing awareness of the important function lipids are now known to play in both the properties and functionality of the brain, such as membrane fluidity and permeability, retrograde signaling, neural plasticity, and neurotransmitter release modulation [6,7]. Studies of blood plasma lipid alteration in psychiatric disorders, in particular, have been numerous over the past decade [8-23], with blood being a relatively easy sample type to procure for studies and most relevant for potential future biomarker implementations. Indeed, this area of research shows promise, evidenced by the distinct 
pattern of schizophrenia-associated lipid alterations being reported in the literature. Among other lipid compounds, the lipid classes most robustly associated with the disorder are ether phospholipids (such as plasmanyl-/plasmenyl- phosphatidylcholine and plasmanyl/plasmenyl- phosphatidylethanolamine) $[9,11-14,24]$ and acylcarnitines $[8,10,15,25,26]$. Associations with ether phospholipids and acylcarnitines have also been reported for other psychiatric disorders, such as major depressive disorder [19-21,27]. Additionally, ceramides have been proposed as a potential biomarker of depression [28].

While blood lipid alterations have been shown for psychiatric disorders, their levels are subject to a degree of variability related to sex, age, metabolic health, and, possibly, other unaccounted factors [29,30]. On the other hand, the study of changes over time in lipid levels of the same individual has the advantage of reducing this variability. Previously, several studies have aimed to investigate the association between changes in blood lipidome composition and symptom improvement for schizophrenia [31-33], but the available evidence is limited. In this study, we measured the blood lipid abundances in 92 individuals diagnosed with schizophrenia and undergoing inpatient treatment. The blood plasma was collected at two distinct time points to investigate the associations between symptom improvement and individual changes in blood plasma lipid levels.

\section{Materials and Methods}

\subsection{Patients and Sample Collection}

Included subjects were inpatients recruited from the Mental Health Research Center, Moscow. The subjects were adult (age range 17-43 years) participants with a diagnosis of SCZ ( $n=92 ; 58 \%$ female) according to the ICD-10 criteria. Sample collection was performed at the Neuroimmunology Laboratory of the Mental Health Research Center, Moscow. Patient evaluation was performed by board-certified psychiatrists of the same center. Clinical symptoms of patients were assessed by Positive and Negative Syndrome Scale (PANSS) [34], a widely used medical scale of symptom severity in schizophrenia. The PANSS score is composed of three components: the positive (e.g., hallucinations and delusions), negative (represented by loss of normal functions), and general psychopathology components. Higher PANSS score values correspond to more severe symptom manifestations. The study was approved by the local ethics committee of the Mental Health Research Centre. Informed consent was obtained from all participants. The entire study was conducted in line with the World Medical Association Declaration of Helsinki formulating ethical principles for medical research involving human subjects.

Plasma was obtained from peripheral venous blood in the morning from individuals that underwent an overnight fast. Plasma samples were collected in $4 \mathrm{~mL}$ Vacutainer tubes containing the chelating agent ethylenediaminetetraacetic acid (EDTA) (BD Vacutainer, Franklin Lakes, NJ, USA). Tubes were centrifuged at $4^{\circ} \mathrm{C}$ at $1100 \times g$ for $15 \mathrm{~min}$. The supernatant was stored immediately in $500 \mu \mathrm{L}$ aliquots at $-80^{\circ} \mathrm{C}$.

\subsection{Lipid Extraction and Lipidomics Measurements}

A mixture of isotopically labeled lipids (SPLASH LIPIDOMIX Mass Spec Standard, Avanti Polar Lipids) was added to methyl tert-butyl ether (MTBE) based on 1 vial for $100 \mathrm{~mL}$ of solvent. All tubes and solvents were pre-cooled down to $0{ }^{\circ} \mathrm{C}$ and manipulations carried out on ice. Frozen plasma was thawed on ice for $2 \mathrm{~h}$. Then, cold methanol $(300 \mu \mathrm{L})$ was added to $40 \mu \mathrm{L}$ of sample aliquot and vigorously vortexed for $1 \mathrm{~min}$. Afterward, $1 \mathrm{~mL}$ of cold MTBE with spiked standards was added, and the mixture was sonicated for $10 \mathrm{~min}$, incubated for $40 \mathrm{~min}$ at $4{ }^{\circ} \mathrm{C}$ in a shaker, and then sonicated for another $10 \mathrm{~min}$. Phase separation was induced by adding $250 \mu \mathrm{L}$ of MS-grade water. Extract was vortexed for $1 \mathrm{~min}$ at $4{ }^{\circ} \mathrm{C}$, then centrifuged for $10 \mathrm{~min}$ at $13,000 \mathrm{rpm}$ and $4{ }^{\circ} \mathrm{C}$. A total of $1000 \mu \mathrm{L}$ of the upper layer, containing most of the lipids, was collected in a fresh Eppendorf tube. Four hundred microliters of re-extraction buffer (MeOH:MTBE: $\mathrm{H}_{2} \mathrm{O}=3: 10: 2.5$ ) was added to the lower phase. Samples were vortexed and centrifuged for $10 \mathrm{~min}$ at 13,000 rpm and $4{ }^{\circ} \mathrm{C}$. Additional $300 \mu \mathrm{L}$ of upper phase was collected, and combined organic phases were 
evaporated to dryness in a Speed Vac concentrator at $30^{\circ} \mathrm{C}$. Dried lipid pellets were stored at $-80{ }^{\circ} \mathrm{C}$ before analysis. The pellets were resuspended in $200 \mu \mathrm{L}$ ice-cold acetonitrileisopropanol mixture $(7: 3(v: v))$. After brief vigorous vortexing, the samples were shaken for $10 \mathrm{~min}$, sonicated in an ice-cooled sonication bath for $10 \mathrm{~min}$, and centrifuged $5 \mathrm{~min}$ at 13,000 rpm. For mass spectrometry analysis, samples were diluted 1:5 and 1:2 with acetonitrile-isopropanol (7:3 (v:v)) for positive and negative ionization modes, respectively.

The liquid chromatography/mass spectrometry system consisted of a Waters Acquity UPLC system (Waters, Manchester, UK) and a Q Exactive orbitrap mass spectrometer (Thermo Fisher Scientific, USA) equipped with a heated electrospray ionization (HESI) probe. Separation of lipids was performed at $60{ }^{\circ} \mathrm{C}$ using a reverse-phase ACQUITY UPLC BEH C8 Column (2.1 × 100 mm, $1.7 \mu \mathrm{m}$, Waters co., Milford, MA, USA) coupled to Vanguard precolumn at a flow rate of $0.4 \mathrm{~mL} / \mathrm{min}$. The mobile phases consisted of water containing $10 \mathrm{mM}$ ammonium acetate (Buffer $\mathrm{A}$ ), and a mixture of acetonitrile and isopropanol (7:3) containing $10 \mathrm{mM}$ ammonium acetate (Buffer B). Both buffers contained $0.1 \%$ formic (positive mode) or acetic acid (negative mode) by volume. Separation was carried out by gradient elution according to the following profile: $1 \mathrm{~min} 55 \% \mathrm{~B}, 3 \mathrm{~min}$ linear gradient from $55 \%$ to $80 \%$ B, 8 min linear gradient from $80 \%$ B to $85 \%$ B, and 3 min linear gradient from $85 \%$ B to $100 \%$ B. After 4.5 min washing with $100 \%$ B, the column was re-equilibrated with $55 \%$ B for $4.5 \mathrm{~min}$. The injection volume was $3 \mu \mathrm{L}$. Mass spectra were acquired both in positive and negative modes in different experiments with a mass range of $m / z$ 100-1500 at a mass resolving power of 70,000 at $m / z$ 200, AGC target: 1E6, Maximum IT: $100 \mathrm{~ms}$. Ion source for scan mode was operated with following parameters: capillary temperature, $250{ }^{\circ} \mathrm{C}$; aux gas heater temperature, $350{ }^{\circ} \mathrm{C}$; spray voltage, $4.5 \mathrm{kV}$; S-lens $\mathrm{RF}$ level, 70; sheath gas $\left(\mathrm{N}_{2}\right)$ flow rate, 45 arbitrary units (a.u.); auxiliary gas $\left(\mathrm{N}_{2}\right)$ flow rate, 20 a.u.; sweep gas $\left(\mathrm{N}_{2}\right)$ flow rate, 4 a.u. Data were acquired on the profile mode. External mass calibration (Pierce LTQ Velos ESI Positive Ion Calibration Solution, Pierce Negative Ion Calibration Solution) without the use of the specific lock masses was employed.

Additional experimental samples were analyzed together with the plasma samples. Empty tubes without plasma (extractions blanks) were placed in the end of the experiment and subjected to the same analysis steps as plasma samples. Quality control (QC) samples were incorporated every 12th position to account for sample preparation and measurement variability. QC samples consisted of aliquots of plasma, mixed before extraction.

For lipid structure elucidation, mass spectra were acquired in data-dependent acquisition mode with active inclusion list. Parameters for full MS scan mode were set as follows: resolution: 70,000 at $\mathrm{m} / \mathrm{z}$ 200; AGC target: 5E5, IT: $50 \mathrm{~ms}$, mass range: 200-1800 for both polarities. All ions from the inclusion list within $10 \mathrm{ppm}$ range were subjected to fragmentation with the following parameters: AGC target: 5E4; IT: $100 \mathrm{~ms}$. Intensity threshold was kept at 8E2 and isolation width was set at 1.2 Da. Stepped normalized collision energy was set at 15\%, 20\%, 25\%, and 30\%. Data were acquired on the profile mode, peptide match option was off, and isotope exclusion was set to on. Acquired spectra were manually curated and identifications were based on MS2 spectra, with MS1 mass error of $<5$ ppm and MS2 mass error of $<8 \mathrm{ppm}$.

\subsection{Lipidomics Data Processing}

Spectra were processed using XCMS software [35], using the "centWave" method for peak detection, the "obiwarp" method for retention time correction, and the "fill-Peaks" method for missing value imputation. Duplicated features were deleted, and isotopes were removed as well, using an in-house algorithm. Abundance values were log2 transformed. Missing values as reported by XCMS were replaced by random values sampled from a normal distribution with mean 11.7 and standard deviation 0.25 , which corresponds to the approximate noise level seen in the experiment. Batch effect was corrected for all samples, including QC and blank samples, by subtracting the median batch value, calculated excluding the QC and blank samples. The feature abundances were then returned to their original scale by adding the median value of abundances in each batch. Features retaining 
high technical variability after batch correction were removed using QC samples according to the following rule: features with standard deviation across QC samples $>1$ (in $\log 2$ scale) were removed from the analysis. Contaminants were filtered out using extraction blank samples according to the following rule: features for which mean abundance in plasma samples < mean abundance in blank samples +1 (in log2 scale) were removed from the analysis. Features were also removed according to retention time thresholds $0<\mathrm{RT}<19$ for positive ionization mode and $0<\mathrm{RT}<14.5$ for negative ionization mode. Samples were not normalized according to total lipid content.

Lipid features were annotated by mass-to-charge $(\mathrm{m} / \mathrm{z})$ values and retention times with one select adduct per lipid class and $m / z$ threshold of $10 \mathrm{ppm}$. Appropriate retention times were determined based on in-house retention time for previously annotated lipid species, as well as chain length and double bond content of the annotated lipid species. Additional MS2 experiments were performed to validate the annotation for part of the lipid compounds.

For downstream statistical analysis, only annotated lipid compounds were used.

\subsection{Statistical Analysis}

Wilcoxon signed-rank and Mann-Whitney U tests were used with continuity correction.

When restricting patients to those treated with certain medications, all individuals that were administered this medication, among possible other drugs, were used, resulting in overlapping groups of patients.

Median base 2 log-transformed fold-changes (log2 fold-change) between the two time points were calculated as the median values of the individuals' differences in lipid abundances between the second and first time points.

When calculating $p$-values for binomials test for correlation coefficients, the probability of a positive correlation was assumed to be $1 / 2$.

When calculating $p$-values for binomial tests for median $\log 2$ fold-change for the subsets of worst and best responders, the probability of the median $\log 2$ fold-change of the subset of worst responders being larger than 0 was assumed to be $1 / 2$. Likewise, the probability of the median $\log 2$ fold-change of the subset of worst responders being larger than the median $\log 2$ fold-change of the subset of best responders was assumed to be $1 / 2$.

For analysis of variance (ANOVA) and interaction effect on lipid changes between time points, the following ANOVA type I model was used: lipid change $\sim$ sex + age + PANSS change + sex * age + PANSS change * sex + PANSS change * age + PANSS change * sex* age. For ANOVA and interaction effect on lipid abundances at the first time point, the same model was used: lipid abundance at first point $\sim$ sex + age + PANSS change + sex * age + PANSS change * sex + PANSS change* age + PANSS change * sex* age. To calculate the excess of nominally significant $(p<0.05)$ lipids for the different main effects and interactions, permutation test was performed. Sample labels were permutated 100 times for the lipid abundances table, and the number of nominally significant $(p<0.05)$ lipids for the different main effects and interactions was calculated each time. The fraction of permutations for which the random number was equal to or higher than the true number of nominally significant lipids was defined as the permutation $p$-value. The ratio of the median number of nominally significant lipids in the permutations to the true number of nominally significant lipids was used to define the fold excess of nominally significant lipids. Permutation test was used to calculate the enrichment of lipid classes among nominally significant lipids as well. A subsample of lipids of the same size as the number of nominally significant lipids was chosen at random, and the number of lipids from the given lipid class was calculated. The fraction of permutations (from 10,000) for which this random number was equal to or higher than the true number of nominally significant lipids from the given lipid class was used to define the permutation $p$-value. 


\section{Results}

\subsection{Study Setup}

We assessed the abundance of 322 lipid species in the blood plasma of 92 individuals diagnosed with schizophrenia collected at two time points: at the beginning and end of their hospitalization at a psychiatric clinic (37 \pm 19 days; Figure S1). Samples were represented by female and male individuals (58\% female) of age ranges $17-43$ years, and information on medication regiment was collected (Table S1). Symptom severity was assessed by Positive and Negative Syndrome Scale (PANSS) [34] score at the two time points (Table S1). Lipidomics measurements were produced using mass spectrometry coupled with liquid chromatography in negative and positive ionization modes. From the reproducibly quantified lipid features, a set of 322 unique lipid compounds was annotated based on their mass-to-charge, retention time values, and fragmentation patterns (Table S2). Assessed lipid species covered 14 lipid classes and aligned well with expected blood plasma lipidome composition [36] (Figure S2).

\subsection{Association between Changes in Lipid Abundances and Symptom Improvement}

All but one patient displayed symptom improvement from the first to the second time point, demonstrated by the reduction in PANSS scores (Figure 1A). However, the improvement in PANSS scores ranged from -102 to 0 point differences, with the top $25 \%$ best responders displaying PANSS score improvements of -102 to -39 point differences $(n=23)$ and the bottom $25 \%$ worst responders displaying PANSS score improvements of -14 to 0 point differences $(n=24)$ (Figure $1 \mathrm{~A})$. The extent of changes in lipid abundances differed depending on PANSS score improvement. While for worst responders, 22 lipids showed significant changes from first to the second time point (worst-response-associated lipids; Wilcoxon signed-rank test, Benjamini-Hochberg correction FDR 5\%; Figure 1B; Table S2), the effect in best responders was lower (Wilcoxon signed-rank test, no significant lipids at Benjamini-Hochberg FDR 5\% threshold; Figure 1B; Table S2). Accordingly, although the levels of worst-response-associated lipids at baseline were similar for best and worst responders, best responders did not display a statistically significant increase from first to the second point (respectively: Mann-Whitney U test, 1 of $22 p<0.05$; Wilcoxon signedrank test, all $22 p>0.05$; Figure 1C,D; Table S2). Among the 22 worst-response-associated lipids, 20 were triglycerides, $30 \%$ of total triglycerides (Figure 1E). Triglycerides with lower carbon number (40-48 carbons in fatty acid residues) were most affected (Figure 1F). These triglycerides were also among the least abundant ones (Figure S3).

The increase in worst-response-associated lipids was not restricted to $25 \%$ of patients displaying the least improvement in PANSS scores, as individuals in the interquartile range with less symptom improvement also displayed this increase in lipid abundance (Figure 2A). Correlation analysis between PANSS score reduction and lipid abundance changes yielded 13 nominally significant lipids, including free fatty acids and triglycerides (Spearman correlation, nominal $p<0.05$ ) (Figure 2B), but these lipids did not pass the $5 \%$ threshold after correction for multiple testing (Benjamini-Hochberg correction). 
A

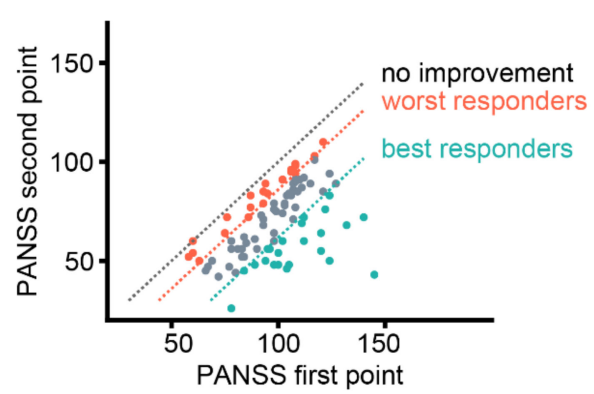

C

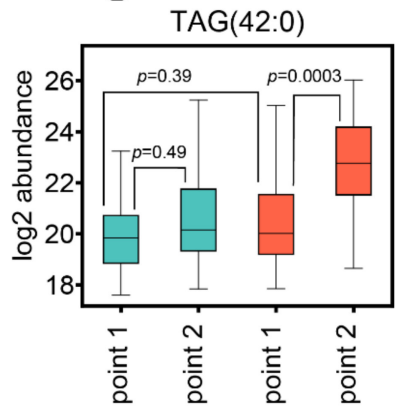

E

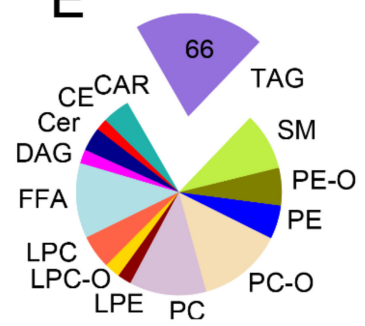

B

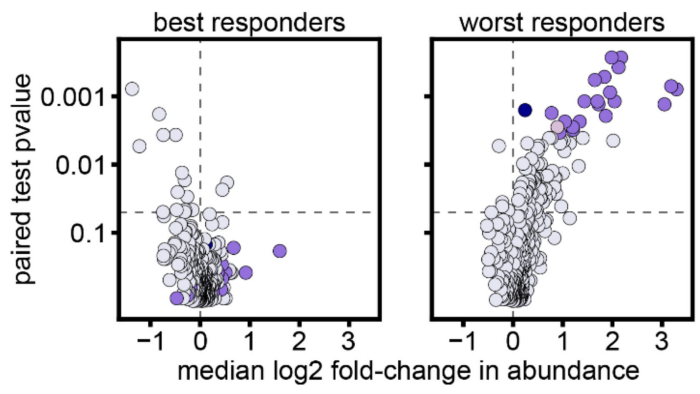

D
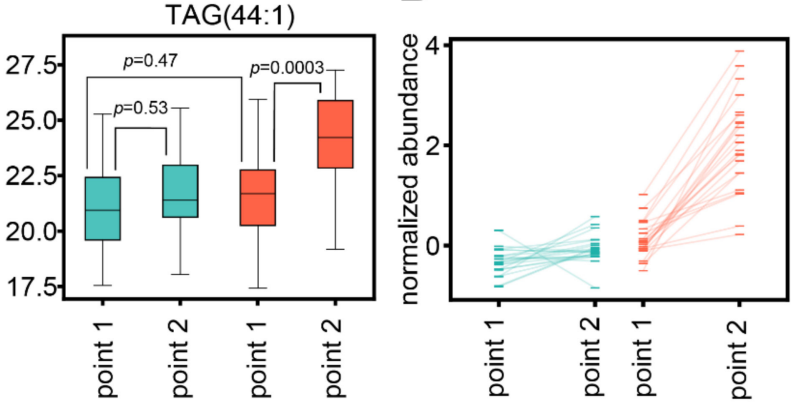

$\mathrm{F}$

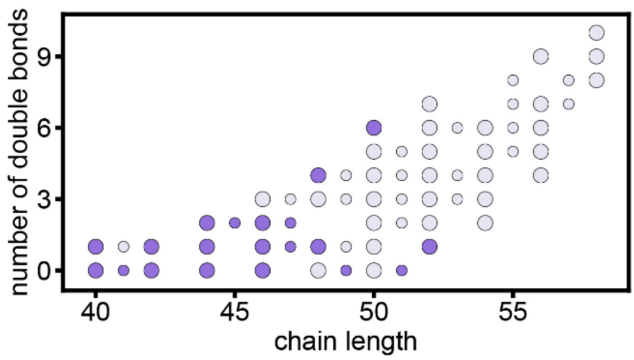

Figure 1. Significant changes in worst responders. (A) PANSS scores at first and second time points for the 92 individuals. Individuals with least improvement in PANSS score (worst responders, $n=24$ ) and most improvement in PANSS score (best responders, $n=23$ ) are marked in orange and green, respectively. Orange and green dashed lines demarcate to the upper and lower quartile of PANSS differences; dashed grey line corresponds to identical PANSS values at the two points. (B) $p$-values of the Wilcoxon signed-rank test plotted against the median base 2 log-transformed fold change (log2 fold-change) between the two time points for individuals with most improvement (left) and least improvement (right). The 22 worst-response-associated lipids are marked in color according to lipid class: TAG, purple; PC, dusty pink; Cer, dark blue. Dashed lines demarcate $\log 2 \mathrm{FC}=0$ and nominal $p=0.05$. (C) $\log 2$ abundances for best and worst responders at first and second time points. Three worst-response-associated lipids with strongest statistical effect $(p<0.0005$ for Wilcoxon signed-tank test for changes in worst responders) are plotted: TAG 42:0, TAG 44:0, TAG 44:1. Noted $p$-values correspond to Wilcoxon signed-rank and Mann-Whitney U test $p$-values for comparisons between groups. Boxplot whiskers and fliers correspond to standard boxplot definition. (D) The median values of the normalized log2 abundances for best and worst responders at first and second time points, for all 22 worst-response-associated lipids. For each lipid, the log2 abundances were normalized by the lipid mean value for all patients at the first time point. (E) Left: the 322 annotated lipids, grouped by lipid class. Right: the 22 worst-response-associated lipids, grouped by lipid class. The numbers of respective triglyceride species are indicated on the plot. (F) The number of carbons in the fatty acid residues (chain length) and number of double bonds for the annotated triglycerides. Worst-response-associated lipids are colored in purple. Larger and smaller circle sizes correspond to even and odd chain triglycerides, respectively. 

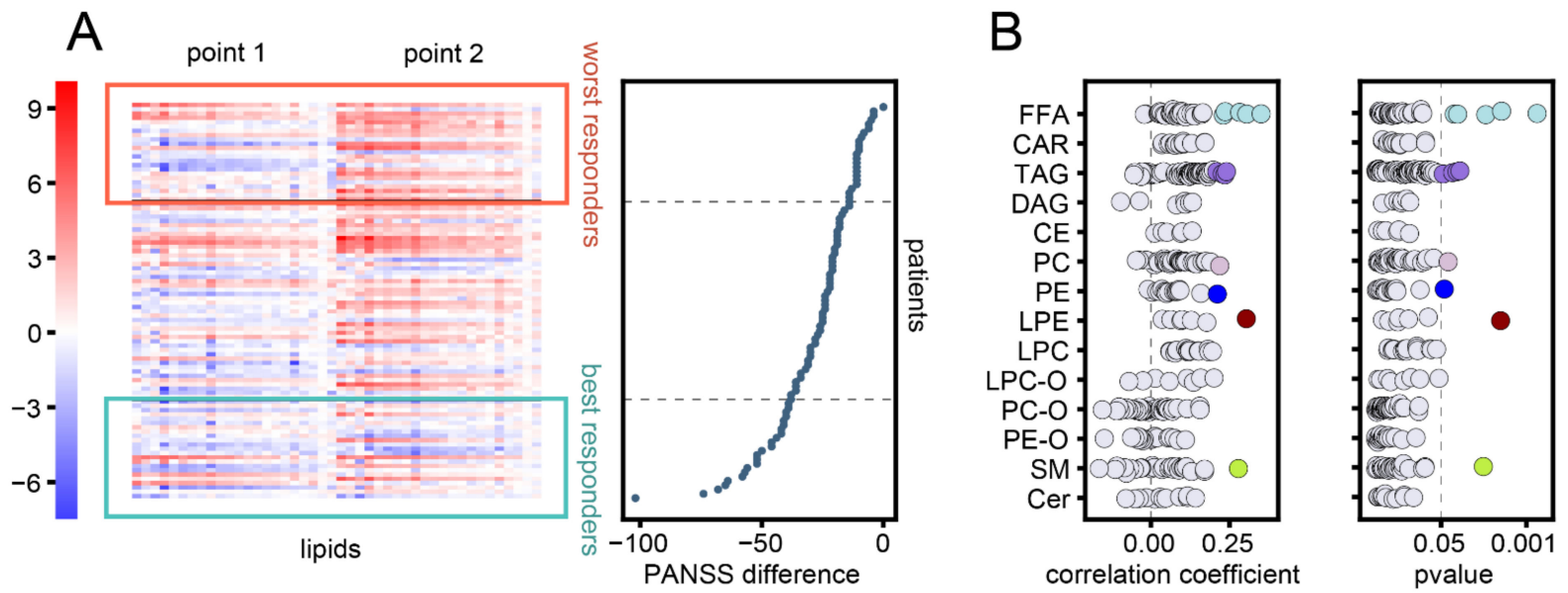

Figure 2. Association between lipid changes and symptom improvement. (A) The normalized base 2 log-transformed ( $\log 2$ ) abundances of the 22 worst-response-associated lipids at first and second time points. For each lipid, the log2 abundances were normalized by the lipid mean value for all patients at the first time point. Individuals were sorted by PANSS difference between the two time points, plotted to the right. Colored boxes and grey horizontal lines delineate the best and worst responders. (B) Spearman correlation coefficients (left) and corresponding $p$-values (right) between changes in lipid abundances and differences in PANSS score. Lipids with nominally significant $p$-values $(<0.05)$ are marked in color. Dashed lines delineate $p$-value 0.05 and correlation coefficient 0 .

\subsection{Influence of Medication}

Because the association between changes in lipid abundance levels and symptom severity could be confounded by medication regimens, we aimed to study this association confined to patients receiving the same drugs. Individuals enrolled in this study received a variety of different medications and often more than one medication at once. In total, 40 different medications were administered to at least one individual, and 15 medications were represented six or more times in the sample population (Figure 3A). We restricted the correlation analysis between symptom improvement and changes in lipid abundance levels to individuals receiving each of these 15 medications. For the three triglyceride species with the strongest worst-response-associated effect ( $p<0.0005$ : TAG 42:0, TAG 44:0, and TAG 44:1), these correlations were positive for most of the medications $(11,13$, and 13 out of the 15 medications for TAG 42:0, TAG 44:0, and TAG 44:1, respectively; Figure 3B), which is significantly more than expected by chance (binomial test $p=0.059,0.0037$, and 0.0037). For the other worst-response-associated lipids, the correlations were positive for $10-15$ of the 15 medications, with $41 \%$ percent of lipids showing significant values and $45 \%$ borderline significant values (binomial test $p<0.05$ and $p=0.059$, respectively; Figure S4).

Similarly, we assessed the changes in lipid abundances between the two time points for best responders and worst responders while restricting the analysis to individuals receiving a particular medication. In total, there were 10 medications for which there were at least two best responders and two worst responders. For TAG 42:0, TAG 44:0, and TAG 44:1, not only were the median lipid abundances increased in worst responders for all of these 10 medications, but for most of them, median changes in worst responders were higher than median changes in best responders $(9,10$, and 9 of the 10 medications for TAG 42:0, TAG 44:0, and TAG 44:1, respectively; binomial test $p=0.0107,0.0001$, and 0.0107; Figure 3C). For these three triglyceride species, quetiapine, fluvoxamine, and diazepam were least associated with symptom severity (Figure 3D). However, quetiapine, fluvoxamine, and diazepam were represented by the smallest number of individuals. Among the medications for which the difference between worst and best responders was strongest, we noted clozapine, chlorpromazine, valproate, and trihexyphenidyl (Figure 3D). For the rest of the worst-response-associated lipids, results were similar, except for haloperidol, for which changes in worst responders were similar to best responders for most of these lipids (Figure 3D). 
A
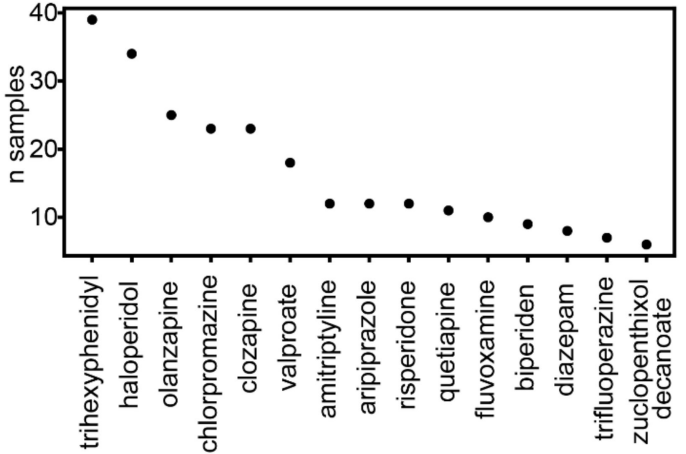

B

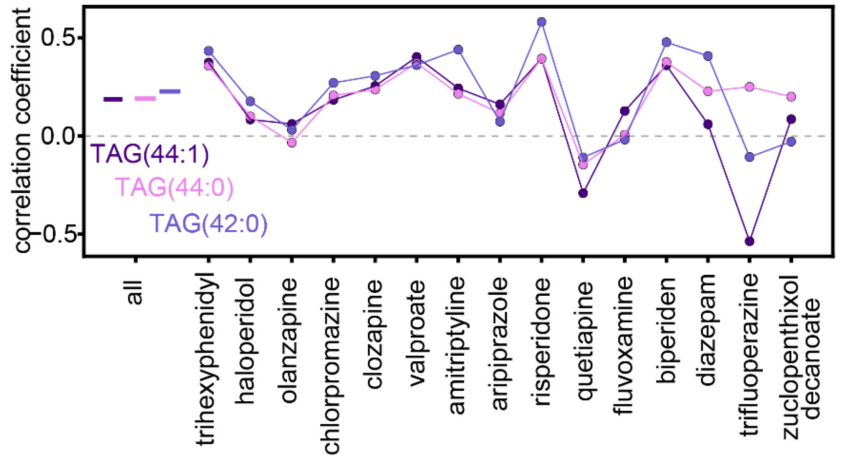

D

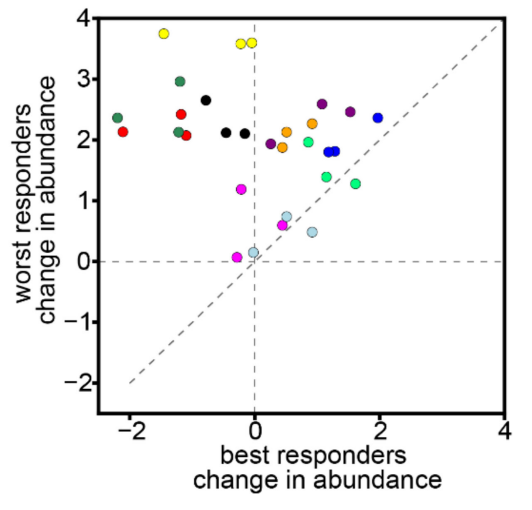

- trihexyphenidyl

- haloperidol

- olanzapine

- chlorpromazine

- clozapine

- valproate

- aripiprazole

- quetiapine

- fluvoxamine

- diazepam
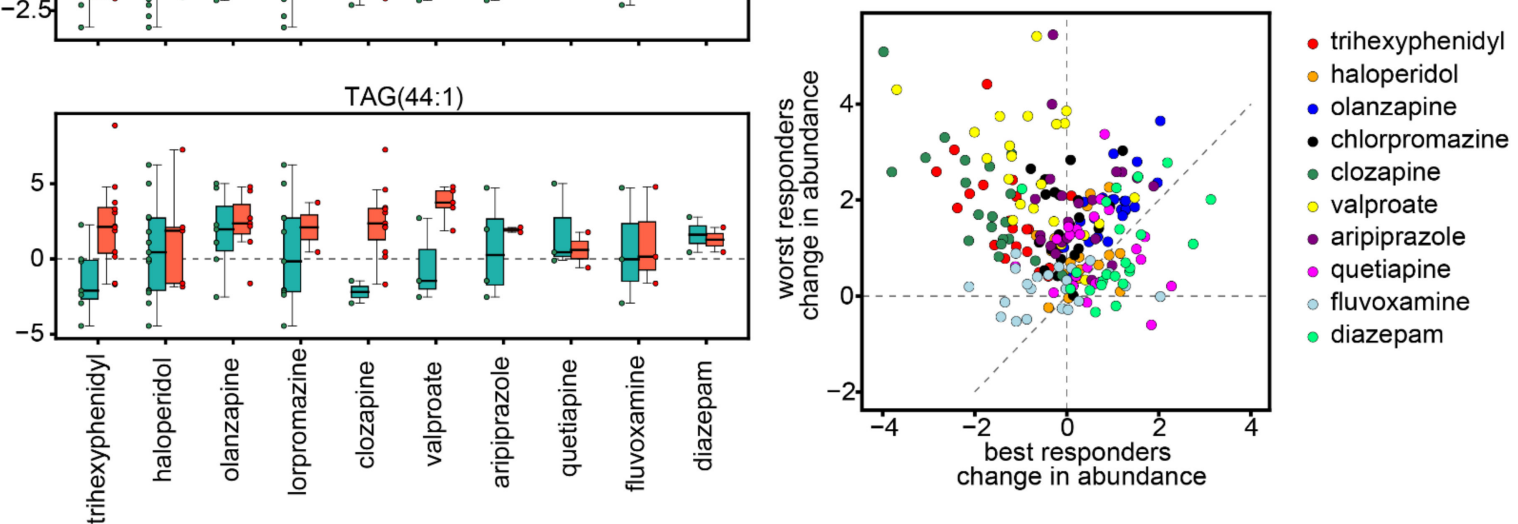

Figure 3. Medication effect. (A) Number of individuals receiving specific medications. The schema includes the medications that were administered to more than 5 individuals. (B) Spearman correlation coefficients between changes in PANSS scores and changes in lipid levels between the two time points, for all patients and only patients receiving a particular medication. Three worst-response-associated lipids with the strongest statistical effect $(p<0.0005$ Wilcoxon signed-rank test for changes in worst responders) are shown: TAG 42:0, TAG 44:0, and TAG 44:1. Different color shades correspond to the three different lipids. The schema includes the medications that were administered to more than 5 individuals. (C) Changes in lipid abundances for best (green) and worst (orange) responders that were receiving a particular medication. Changes in abundances were calculated as the base 2 log-transformed fold-changes (log2FCs) between the time points. The particular triglyceride species are marked at the top. Medications that were administered to at least two worst and two best responders are shown. In addition to the boxplots, abundance changes for each individual are marked as a circle. Boxplot whiskers correspond to the standard boxplot definition. (D) Median changes in lipid abundances for best and worst responders that were receiving a particular medication, colored by medication. Top: TAG 42:0, TAG 44:0, and TAG 44:1. Bottom: all 22 worst-response-associated lipids. Changes in abundances were calculated as the log2FC between the time points. Medications that were administered to at least two worst and two best responders are shown. 


\subsection{Influence of Sex and Age}

While age and time between the two plasma collection points were similar for best and worst responders (Figures S5 and S6), there was a strong sex imbalance. Most of the best responders were female (Figure S7), making sex a potential confounding factor for symptom improvement. Comparison of changes in female best and worst responders demonstrated that female worst responders had increased abundances for worst-responseassociated lipids compared to best responders, as was the case for the best and worst responding individuals of the two sexes (Figure S8). For both female and male individuals, the correlation between PANSS score reduction and changes in lipid abundances for worstresponse-associated lipids was positive for $100 \%$ and $82 \%$ of the lipids (binomial test; $p=2.38 \times 10^{-7}$ and 0.0022; Figure S9), indicating that the observed effect of increased worst-response-associated lipids at the second time point could not be explained by the imbalance in sex distribution observed in the data.

Additionally, we aimed to investigate whether there was an interaction effect between PANSS score changes, sex, and age on changes in lipid abundances between the two time points using analysis of variance (ANOVA). Higher-order interactions between PANSS score changes, sex, and age (PANSS change * sex * age) did not show any detectable statistical effect (ANOVA, 1.28 fold excess of nominally significant lipids at $p<0.05$, permutation $p=0.46$; Figure S10A; Table S2). For PANSS scores changes and sex, as well as PANSS scores and age, there did seem to be a significant interaction effect, though not strong enough to pass the multiple testing correction (ANOVA, 3.8 and 3.9 fold excess of nominally significant lipids at $p<0.05$, permutation $p=0.07$ and $=0.08$, respectively; Figure S10A; Table S2). The nominally significant lipids for the interaction effect PANSS change * sex (nominal $p<0.05$ ) were particularly enriched with ether phosphatidylcholines (PC-O) (permutation $p<0.0001$; Figure S10B), while the nominally significant lipids for interaction effect PANSS change * age (nominal $p<0.05$ ) were enriched with both ether phosphatidylcholines and ether phosphatidylethanolamines (PC-O, PE-O), as well as sphingomyelins (SM) (permutation $p=0.0019,0.017,0.0158$, respectively; Figure S10C).

\subsection{Lipid Profiles at First Time Point}

Unlike changes in lipid abundances between the two time points, the lipid abundances at the first time point could potentially function as actionable predictors of symptom improvement. However, blood plasma lipids are subject to interindividual variability related to various factors, including basic parameters like sex and age [30]. Like others [30], we have found that both sex and age have a strong effect on lipid abundances (number of significant lipids $n=41$ and $n=36$ for sex and age, respectively; ANOVA, BenjaminiHochberg correction FDR 5\%; Figure S11A; Table S2). We did not find statistically significant main effects or interaction effect on the lipid abundances related to the change in PANSS scores (ANOVA, no significant lipids after Benjamini-Hochberg correction FDR 5\%; factors: PANSS change, PANSS change * sex, PANSS change * age; Table S2). However, the interaction effect between PANSS score changes and sex on the lipid abundances was stronger than the other interactions and was also stronger than the main effect of PANSS score changes on lipid abundances (ANOVA for PANSS change * sex: 2.45 fold excess of nominally significant lipids at $p<0.05$, permutation $p=0.15$; ANOVA for PANSS change, PANSS change * age, PANSS change * sex * age: $1.27,0.17,0.27$ fold excess of nominally significant lipids at $p<0.05$, permutation $p=0.45,0.91,0.91$; Figure S11B; Table S2).

Symptom improvement after antipsychotic treatment was shown to be sex-dependent in this study (Figure S7) as well as others [37,38]. For this reason, the association between symptom improvement and lipid abundances at the first point was strongly confounded by sex, which warranted the separate investigation for males and females. Consistent with the stronger PANSS change * sex interaction effect in the analysis of variance, we observed that correlation coefficients between PANSS score changes and lipid abundances at the first time points were inconsistent between females and males (Figure S11C; Table S2). The correlation coefficients of the nominally significant (Spearman correlation nominal $p<0.05$ ) 
lipids had mostly opposite signs for females and males. However, for both females and males, these correlation levels were not statistically significant after correction for multiple testing, and the observed effect would require further confirmations.

\section{Discussion}

In this study, we assessed the abundances of 322 lipids in the blood plasma of 92 patients diagnosed with schizophrenia collected at two points in time during the individuals' inpatient treatment. We aimed to investigate the association between individual changes in lipid abundances and improvement in symptom severity. We found that, for patients with the least improvement in symptom severity, 22 lipids, including 20 triglyceride species, were increased at the second time point, while patients with most improvement did not demonstrate the same increase in lipid levels. The most affected triglycerides contained a lower number of carbons (40-48 carbons in fatty acid residues), while the triglyceride species most abundant in blood plasma were not among the significantly increased lipids.

Others have studied the association between lipid levels and symptom improvement. Levels of total triglycerides, which are more routinely used in clinical practice but provide a rougher approximation of the blood plasma lipidome, have been shown to correlate negatively with PANSS score differences in some studies [39]. This effect, however, was not reproduced by others [39]. In our study, we have found the opposite direction of correlation for triglyceride species. This effect, however, does directly contradict the former studies, since the abundances of triglyceride species can differ in orders of magnitude. Total triglyceride measurements are strongly represented by the top most abundant lipid species, which we did not find to be significantly associated with symptom improvement. In our study, the triglycerides associated with symptom improvement were triglycerides containing a lower number of carbons. Interestingly, one study has found that an increase in triglycerides with these chain length ranges was associated with weight gain in first psychotic episode patients after undergoing treatment [40]. In general, short-chain triglycerides, in particular, have been found to be associated with diabetes and nonalcoholic fatty liver disease [41,42]. These observations suggest that the increase in particular triglycerides we detected in worst responders could be related to metabolic abnormalities induced by antipsychotic treatment, affecting the treatment response of the patients.

Antipsychotic treatment has previously been shown to affect both clinical lipid measurements, such as total triglycerides, and compounds at the level of lipid species [12,25,32,43,44]. Some antipsychotics have been shown to have a larger effect on the blood plasma lipids than others, and their effect on particular compounds was different between them. Therefore, symptom-dependent imbalance in treatment regimen could potentially confound the association between lipid levels and treatment response. Nevertheless, the association between specific triglyceride levels and symptom improvement we report could not be explained by a bias in the treatment regimen, but rather the effect was reproduced for most of the medications independently. Indeed, for most of the medications, changes in lipid levels in patients receiving a particular medication were higher for worst responders compared to best responders. Among the medications for which the difference between worst and best responders was strongest, we noted clozapine (atypical antipsychotic), chlorpromazine (typical antipsychotic), valproate (mood stabilizer), and trihexyphenidyl (antimuscarinic). However, one limitation of the study was the large variety of medications administered to the patients, resulting in patients receiving multiple psychopharmacological drugs simultaneously, and some medications were represented by only a small number of patients.

Consistent with reported differences in treatment response between sexes [37,38], we saw that females demonstrated more improvement in symptom severity after inpatient treatment than males. Nevertheless, the symptom-dependent increase in worst-responseassociated lipids was present for females and males alike. However, the number of males with larger symptom improvement was relatively small, and a significant sex-dependent association could be present but not detected in our analysis. We did find an enrichment of 
ether phosphatidylcholines (PC-O) among the nominally significant lipids for the interaction effect between changes in PANSS scores and sex on the changes in lipid abundances. However, the differing ranges of symptom improvement for females and males call for a more detailed investigation of the potential sex-specific effects, possibly with larger sample sizes.

While lipid abundances at the first time point could have been considered as predictors of future symptom improvement, we did not find a significant association between these abundances and the PANSS score changes. We did find that these associations were different for females and males, with the respective correlations showing opposite directions. However, these effects were not particularly strong and were not statistically significant after correction for multiple testing, warranting further investigation.

While there is ample evidence of metabolic abnormalities linked to schizophrenia and other psychiatric disorders, such as diabetes, cardiovascular disorder, and weight gain [45-49], it is still unclear whether these abnormalities co-occur with schizophrenia because of side effects from medications, lifestyle and diet, certain genetic predispositions, or possibly negative physiological impact of the psychiatric disorder. In this study, we have likewise found a metabolic signature associated with the severity of schizophrenia manifestation, which could also be linked to metabolic alterations found in common metabolic disorders. We have also found some weaker evidence for possible differences in the association of symptom improvement and lipid response between females and males. More studies are needed to recognize the implications of such findings for the treatment and understanding of the pathophysiology of schizophrenia.

Supplementary Materials: The following are available online at https: / /www.mdpi.com/article / 10.3390/biom11050720/s1, Figures S1-S11, Table S1: sample information, Tables S1 and S2: lipid annotation and statistical results.

Author Contributions: A.B. was involved in patient recruitment. T.K. and S.Z. collected the samples. E.S. and N.A. performed lipidomics experiments. A.T. and D.P analyzed data. A.T. and D.P. wrote the manuscript. All authors have read and agreed to the published version of the manuscript.

Funding: This work was supported by the Russian Science Foundation under grant 19-74-00151.

Institutional Review Board Statement: The study was approved by the local ethics committee of the Mental Health Research Centre. The entire study was conducted in line with the World Medical Association Declaration of Helsinki formulating ethical principles for medical research involving human subjects.

Informed Consent Statement: Informed consent was obtained from all subjects involved in the study.

Data Availability Statement: The data are available from the corresponding author upon reasonable request.

Acknowledgments: We thank Philipp Khaitovich for helpful discussions and comments on the manuscript.

Conflicts of Interest: The authors declare no conflict of interest.

\author{
Abbreviations \\ DAG diacylglycerol \\ TAG triacylglycerol \\ FFA free fatty acid \\ CAR acylcarnitine \\ CE cholesteryl ester \\ PC phosphatidylcholine \\ PC-O plasmanyl-/plasmenyl-phosphatidylcholine \\ LPC lysophosphatidylcholine
}


LPC-O lyso- plasmanyl-/plasmenyl- phosphatidylcholine

PE phosphatidylethanolamine

PE-O plasmanyl-/plasmenyl- phosphatidylethanolamine

LPE lysophosphatidylethanolamine

Cer ceramide

SM sphingomyelin

\section{References}

1. McCutcheon, R.A.; Pillinger, T.; Mizuno, Y.; Montgomery, A.; Pandian, H.; Vano, L.; Marques, T.R.; Howes, O.D. The efficacy and heterogeneity of antipsychotic response in schizophrenia: A meta-analysis. Mol. Psychiatry 2019, 1-11. [CrossRef] [PubMed]

2. Potkin, S.G.; Kane, J.M.; Correll, C.U.; Lindenmayer, J.P.; Agid, O.; Marder, S.R.; Olfson, M.; Howes, O.D. The neurobiology of treatment-resistant schizophrenia: Paths to antipsychotic resistance and a roadmap for future research. NPJ Schizophr. 2020, 6, 1-10. [CrossRef] [PubMed]

3. Fond, G.; d'Albis, M.A.; Jamain, S.; Tamouza, R.; Arango, C. The promise of biological markers for treatment response in first-episode psychosis: A systematic review. Schizophr. Bull. 2015, 41, 559-573. [CrossRef] [PubMed]

4. Wenk, M.R. The emerging field of lipidomics. Nat. Rev. Drug Discov. 2005, 4, 594-610. [CrossRef] [PubMed]

5. O'Donnell, V.B.; Ekroos, K.; Liebisch, G.; Wakelam, M. Lipidomics: Current state of the art in a fast moving field. Wiley Interdiscip. Rev. Syst. Biol. Med. 2020, 12, e1466. [CrossRef] [PubMed]

6. Piomelli, D.; Astarita, G.; Rapaka, R. A neuroscientist's guide to lipidomics. Nat. Rev. Neurosci. 2007, 8, 743-754. [CrossRef] [PubMed]

7. Lauwers, E.; Goodchild, R.; Verstreken, P. Membrane Lipids in Presynaptic Function and Disease. Neuron 2016, 90, 11-25. [CrossRef]

8. Cao, B.; Wang, D.; Pan, Z.; Brietzke, E.; McIntyre, R.S.; Musial, N.; Mansur, R.B.; Subramanieapillai, M.; Zeng, J.; Huang, N.; et al. Characterizing acyl-carnitine biosignatures for schizophrenia: A longitudinal pre- and post-treatment study. Transl. Psychiatry 2019, 9, 1-13. [CrossRef]

9. Wood, P.L.; Unfried, G.; Whitehead, W.; Phillipps, A.; Wood, J.A. Dysfunctional plasmalogen dynamics in the plasma and platelets of patients with schizophrenia. Schizophr. Res. 2015, 161, 506-510. [CrossRef]

10. Kriisa, K.; Leppik, L.; Balõtšev, R.; Ottas, A.; Soomets, U.; Koido, K.; Volke, V.; Innos, J.; Haring, L.; Vasar, E.; et al. Profiling of acylcarnitines in first episode psychosis before and after antipsychotic treatment. J. Proteome Res. 2017, 1, 3558-3566. [CrossRef]

11. Leppik, L.; Parksepp, M.; Janno, S.; Koido, K.; Haring, L.; Vasar, E.; Zilmer, M. Profiling of lipidomics before and after antipsychotic treatment in first-episode psychosis. Eur. Arch. Psychiatry Clin. Neurosci. 2020, 270, 59-70. [CrossRef]

12. Yan, L.; Zhou, J.; Wang, D.; Si, D.; Liu, Y.; Zhong, L.; Yin, Y. Unbiased lipidomic profiling reveals metabolomic changes during the onset and antipsychotics treatment of schizophrenia disease. Metabolomics 2018, 14, 1-13. [CrossRef]

13. Wang, D.; Cheng, S.L.; Fei, Q.; Gu, H.; Raftery, D.; Cao, B.; Sun, X.; Yan, J.; Zhang, C.; Wang, J. Metabolic profiling identifies phospholipids as potential serum biomarkers for schizophrenia. Psychiatry Res. 2019, 272, 18-29. [CrossRef]

14. Wang, D.; Sun, X.; Maziade, M.; Mao, W.; Zhang, C.; Wang, J.; Cao, B. Characterising phospholipids and free fatty acids in patients with schizophrenia: A case-control study. World J. Biol. Psychiatry 2020, 1-14. [CrossRef]

15. Cao, B.; Wang, D.; Pan, Z.; McIntyre, R.S.; Brietzke, E.; Subramanieapillai, M.; Nozari, Y.; Wang, J. Metabolic profiling for water-soluble metabolites in patients with schizophrenia and healthy controls in a Chinese population: A case-control study. World J. Biol. Psychiatry 2020, 21, 357-367. [CrossRef]

16. He, Y.; Yu, Z.; Giegling, I.; Xie, L.; Hartmann, A.M.; Prehn, C.; Adamski, J.; Kahn, R.; Li, Y.; Illig, T.; et al. Schizophrenia shows a unique metabolomics signature in plasma. Transl. Psychiatry 2012, 2, e149. [CrossRef]

17. Brunkhorst-Kanaan, N.; Klatt-Schreiner, K.; Hackel, J.; Schröter, K.; Trautmann, S.; Hahnefeld, L.; Wicker, S.; Reif, A.; Thomas, D.; Geisslinger, G.; et al. Targeted lipidomics reveal derangement of ceramides in major depression and bipolar disorder. Metabolis 2019, 95, 65-76. [CrossRef]

18. Gracia-Garcia, P.; Rao, V.; Haughey, N.J.; Ratnam Banduru, V.V.; Smith, G.; Rosenberg, P.B.; Lobo, A.; Lyketsos, C.G.; Mielke, M.M. Elevated plasma ceramides in depression. J. Neuropsychiatry Clin. Neurosci. 2011, 23, 215-218. [CrossRef]

19. Liu, X.; Li, J.; Zheng, P.; Zhao, X.; Zhou, C.; Hu, C.; Hou, X.; Wang, H.; Xie, P.; Xu, G. Plasma lipidomics reveals potential lipid markers of major depressive disorder. Anal. Bioanal. Chem. 2016, 408, 6497-6507. [CrossRef]

20. Liu, X.; Zheng, P.; Zhao, X.; Zhang, Y.; Hu, C.; Li, J.; Zhao, J.; Zhou, J.; Xie, P.; Xu, G. Discovery and validation of plasma biomarkers for major depressive disorder classification based on liquid chromatography-mass spectrometry. J. Proteome Res. 2015, 14, 2322-2330. [CrossRef]

21. Demirkan, A.; Isaacs, A.; Ugocsai, P.; Liebisch, G.; Struchalin, M.; Rudan, I.; Wilson, J.F.; Pramstaller, P.P.; Gyllensten, U.; Campbell, H.; et al. Plasma phosphatidylcholine and sphingomyelin concentrations are associated with depression and anxiety symptoms in a Dutch family-based lipidomics study. J. Psychiatr. Res. 2013, 47, 357-362. [CrossRef]

22. Cai, H.; Cao, T.; Li, N.; Fang, P.; Xu, P.; Wu, X.; Zhang, B.; Xiang, D. Quantitative monitoring of a panel of stress-induced biomarkers in human plasma by liquid chromatography-tandem mass spectrometry: An application in a comparative study between depressive patients and healthy subjects. Anal. Bioanal. Chem. 2019, 411, 5765-5777. [CrossRef] 
23. Kim, E.Y.; Lee, J.W.; Lee, M.Y.; Kim, S.H.; Mok, H.J.; Ha, K.; Ahn, Y.M.; Kim, K.P. Serum lipidomic analysis for the discovery of biomarkers for major depressive disorder in drug-free patients. Psychiatry Res. 2018, 265, 174-182. [CrossRef]

24. Kaddurah-Daouk, R.; McEvoy, J.; Baillie, R.; Zhu, H.; Yao, J.K.; Nimgaonkar, V.L.; Buckley, P.F.; Keshavan, M.S.; Georgiades, A.; Nasrallah, H.A. Impaired plasmalogens in patients with schizophrenia. Psychiatry Res. 2012, 198, 347-352. [CrossRef]

25. Cao, B.; Jin, M.; Brietzke, E.; McIntyre, R.S.; Wang, D.; Rosenblat, J.D.; Ragguett, R.M.; Zhang, C.; Sun, X.; Rong, C.; et al. Serum metabolic profiling using small molecular water-soluble metabolites in individuals with schizophrenia: A longitudinal study using a pre-post-treatment design. Psychiatry Clin. Neurosci. 2019, 73, 100-108. [CrossRef]

26. Cuturic, M.; Abramson, R.K.; Breen, R.J.; Edwards, A.C.; Levy, E.E. Comparison of serum carnitine levels and clinical correlates between outpatients and acutely hospitalised individuals with bipolar disorder and schizophrenia: A cross-sectional study. World J. Biol. Psychiatry 2016, 17, 475-479. [CrossRef]

27. Knowles, E.E.; Huynh, K.; Meikle, P.J.; Göring, H.H.H.; Olvera, R.L.; Mathias, S.R.; Duggirala, R.; Almasy, L.; Blangero, J.; Curran, J.E.; et al. The lipidome in major depressive disorder: Shared genetic influence for ether-phosphatidylcholines, a plasma-based phenotype related to inflammation, and disease risk. Eur. Psychiatry 2017, 43, 44-50. [CrossRef] [PubMed]

28. Dinoff, A.; Herrmann, N.; Lanctôt, K.L. Ceramides and depression: A systematic review. J. Affect. Disord. 2017, 213, 35-43. [CrossRef] [PubMed]

29. Wong, M.W.K.; Braidy, N.; Pickford, R.; Vafaee, F.; Crawford, J.; Muenchhoff, J.; Schofield, P.; Attia, J.; Brodaty, H.; Sachdev, P.; et al. Plasma lipidome variation during the second half of the human lifespan is associated with age and sex but minimally with BMI. PLoS ONE 2019, 14, e0214141. [CrossRef] [PubMed]

30. Beyene, H.B.; Olshansky, G.; Smith, A.A.T.; Giles, C.; Huynh, K.; Cinel, M.; Mellett, N.A.; Cadby, G.; Hung, J.; Hui, J.; et al. High-coverage plasma lipidomics reveals novel sex-specific lipidomic fingerprints of age and BMI: Evidence from two large population cohort studies. PLoS Biol. 2020, 18, e3000870. [CrossRef] [PubMed]

31. Chen, Y.L.; Chen, K.P.; Chiu, C.C.; Tai, M.H.; Lung, F.W. Early predictors of poor treatment response in patients with schizophrenia treated with atypical antipsychotics. BMC Psychiatry 2018, 18, 1-8. [CrossRef]

32. De Almeida, V.; Alexandrino, G.L.; Aquino, A.; Gomes, A.F.; Murgu, M.; Dobrowolny, H.; Guest, P.C.; Steiner, J.; Martins-de-Souza, D. Changes in the blood plasma lipidome associated with effective or poor response to atypical antipsychotic treatments in schizophrenia patients. Prog. Neuro Psychopharmacol. Biol. Psychiatry 2020, 101, 109945. [CrossRef]

33. Tessier, C.; Sweers, K.; Frajerman, A.; Bergaoui, H.; Ferreri, F.; Delva, C.; Lapidus, N.; Lamaziere, A.; Roiser, J.P.; De Hert, M.; et al. Membrane lipidomics in schizophrenia patients: A correlational study with clinical and cognitive manifestations. Transl. Psychiatry 2016, 6, e906. [CrossRef]

34. Kay, S.R.; Fiszbein, A.; Opler, L.A. The positive and negative syndrome scale (PANSS) for schizophrenia. Schizophr. Bull. 1987, 13, 261. [CrossRef]

35. Smith, C.A.; Want, E.J.; O’Maille, G.; Abagyan, R.; Siuzdak, G. XCMS: Processing mass spectrometry data for metabolite profiling using nonlinear peak alignment, matching, and identification. Anal. Chem. 2006, 78, 779-787. [CrossRef]

36. Burla, B.; Arita, M.; Arita, M.; Bendt, A.K.; Cazenave-Gassiot, A.; Dennis, E.A.; Ekroos, K.; Han, X.; Ikeda, K.; Liebisch, G.; et al. MS-based lipidomics of human blood plasma: A community-initiated position paper to develop accepted guidelines. J. Lipid Res. 2018, 59, 2001-2017. [CrossRef]

37. Szymanski, S.; Lieberman, J.A.; Alvir, J.M.; Mayerhoff, D.; Loebel, A.; Geisler, S.; Chakos, M.; Koreen, A.; Jody, D.; Kane, J.; et al. Gender differences in onset of illness, treatment response, course, and biologic indexes in first-episode schizophrenic patients. Am. J. Psychiatry 1995, 152(5), 698-703. [CrossRef]

38. Zhang, X.Y.; Xiu, M.H.; De Yang, F.; Haile, C.N.; Kosten, T.A.; Kosten, T.R. Gender differences in never-medicated first-episode schizophrenia and medicated chronic schizophrenia patients. J. Clin. Psychiatry 2012, 73, 1025-1033. [CrossRef]

39. Kim, D.D.; Barr, A.M.; Fredrikson, D.H.; Honer, W.G.; Procyshyn, R.M. Association between Serum Lipids and Antipsychotic Response in Schizophrenia. Curr. Neuropharmacol. 2019, 17, 852-860. [CrossRef]

40. Suvitaival, T.; Mantere, O.; Kieseppä, T.; Mattila, I.; Pöhö, P.; Hyötyläinen, T.; Suvisaari, J.; Orešič, M. Serum metabolite profile associates with the development of metabolic co-morbidities in first-episode psychosis. Transl. Psychiatry 2016, 6, e951. [CrossRef]

41. Rhee, E.P.; Cheng, S.; Larson, M.G.; Walford, G.A.; Lewis, G.D.; McCabe, E.; Yang, E.; Farrell, L.; Fox, C.S.; O’Donnell, C.J.; et al. Lipid profiling identifies a triacylglycerol signature of insulin resistance and improves diabetes prediction in humans. J. Clin. Investig. 2011, 121, 1402-1411. [CrossRef]

42. Orešič, M.; Hyötyläinen, T.; Kotronen, A.; Gopalacharyulu, P.; Nygren, H.; Arola, J.; Castillo, S.; Mattila, I.; Hakkarainen, A.; Borra, R.J.; et al. Prediction of non-alcoholic fatty-liver disease and liver fat content by serum molecular lipids. Diabetologia 2013, 56, 2266-2274. [CrossRef]

43. Li, R.; Zhang, Y.; Zhu, W.; Ding, C.; Dai, W.; Su, X.; Dai, W.; Xiao, J.; Xing, Z.; Huang, X. Effects of olanzapine treatment on lipid profiles in patients with schizophrenia: A systematic review and meta-analysis. Sci. Rep. 2020, 10, 1-14. [CrossRef]

44. Meyer, J.M.; Koro, C.E. The effects of antipsychotic therapy on serum lipids: A comprehensive review. Schizophr. Res. 2004, 70, 1-17. [CrossRef]

45. Mitchell, A.J.; Vancampfort, D.; Sweers, K.; Van Winkel, R.; Yu, W.; De Hert, M. Prevalence of metabolic syndrome and metabolic abnormalities in schizophrenia and related disorders-a systematic review and meta-analysis. Schizophr. Bull. 2013, 39, 306-318. [CrossRef] 
46. Penninx, B.W.J.H.; Lange, S.M.M. Metabolic syndrome in psychiatric patients: Overview, mechanisms, and implications. Dialogues Clin. Neurosci. 2018, 20, 63. [CrossRef]

47. Hert, M.D.; Dekker, J.M.; Wood, D.; Kahl, K.G.; Möller, H.J. Cardiovascular disease and diabetes in people with severe mental illness position statement from the European Psychiatric Association (EPA), supported by the European Association for the Study of Diabetes (EASD) and the European Society of Cardiology ESC. Eur. Psychiatry 2009, 2, 49-59.

48. Sartorius, N. Depression and diabetes. Dialogues Clin. Neurosci. 2018, 20, 47. [CrossRef]

49. Zhang, J.P.; Lencz, T.; Zhang, R.X.; Nitta, M.; Maayan, L.; John, M.; Robinson, D.G.; Fleischhacker, W.W.; Kahn, R.S.; Ophoff, R.A.; et al. Pharmacogenetic Associations of Antipsychotic Drug-Related Weight Gain: A Systematic Review and Meta-analysis. Schizophr. Bull. 2016, 42, 1418-1437. [CrossRef] 